\title{
COST-EFFECTIVENESS OF ORPHAN DRUGS FOR LEUKEMIA TREATMENT: A SYSTEMATIC REVIEW
}

\author{
Nguyen Do Hong Nhung ${ }^{*}$, Tran Thi Ngoc Van*, Hoang Thy Nhac Vu*
}

\section{ABSTRACT}

Introduction: Leukemia is a rare disease related to hematologic cancer stemming from the bone marrow. The Vietnam Ministry of Health $(\mathrm{MOH})$ promulgated the Orphan Drugs List, in which there were 37 orphan drugs indicated for leukemia. This study aimed to systematically review all studies on the cost-effectiveness analysis (CEA) of these orphan drugs in leukemia treatment. Materials and methods: This study conducted a systematic review on all studies published till August 2021 on the National Center for Biotechnology Information (NCBI), SpringerLink, and Biomed Central. 23 studies were selected in this systematic review, which were studies that had available full-texts, were written in English, aimed to analyze the costeffectiveness of leukemia drugs listed on the Vietnam MOH's Orphan Drugs List. The results were presented by describing CEA findings by five different leukemia types, with a focus on the incremental cost-effectiveness ratio (ICER) of each orphan drug and the comparison to the willingness-to-pay (WTP) threshold. All the cost currency values were converted to USD in 2021 to make comparison. Results: Of 37 drugs on the Vietnam MOH's orphan drug list, 24 drugs were cost-effectiveness analyzed with available fulltexts. Of 23 selected studies in the review, there were 10 studies regarding lymphocytic leukemia $(43.5 \%)$ and 13 studies regarding myeloid leukemia $(56.5 \%) .60 .9 \%$ studied on

*University of Medicine and Pharmacy, Ho Chi Minh City

Responsible person: Hoang Thy Nhac $\mathrm{Vu}$

Email: hoangthynhacvu@ump.edu.vn

Date of receipt: 29/6/2021

Date of scientific judgment: 30/7/2021

Reviewed date: $20 / 8 / 2021$ relapsed/refractory patients, $39.1 \%$ conducted cost-analyses with a social perspective, and $47.7 \%$ used overall survival combined with progression-free survival (OS-PFS) as a clinical endpoint. Quality-adjusted life year (QALY) was used as an effectiveness measurement unit in $65.2 \%$ of the total selected studies. 15 studies made a conclusion that their studied orphan drugs were cost-effective. $100 \%$ of the studies regarding acute lymphoblastic leukemia showed the orphan drugs of interest were cost-effective (ICER < WTP threshold); while about $50 \%$ of the studies regarding other leukemia types showed the orphan drugs of interest were not costeffective (ICER > WTP threshold) and thus required suitable financial aid. Conclusion: This study provided information on the costeffectiveness of 24 out of 37 orphan drugs for leukemia treatment listed on the Vietnam MOH's Orphan Drugs List. These orphan drugs could be considered as a financial burden for leukemia patients and other potential payers such as the Vietnam Social Security due to their considerably high cost.

\section{INTRODUCTION}

Leukemia is a rare disease related to hematologic cancer stemming from the bone marrow. Regarding the World Health Organization (WHO), more than 474,000 leukemia cases were newly diagnosed in 2020 worldwide, in which, the number of leukemia cases and its mortality rate in Asian countries ranked first globally (49\% and $54 \%$, respectively) ${ }^{[1]}$.

Several therapies have been used to control leukemia, including chemotherapy, biological therapy, radiation therapy, targeted 
therapy, and stem cell transplant, in which most drugs are classified as orphan drugs with considerably high costs. In 2019, the Vietnam Ministry of Health (MOH) promulgated the Orphan Drugs List, in which there were 37 drugs used for leukemia treatment. This list provided a basis for assessing and prioritizing the issuance of circulation dossiers for these drugs, as well as increase patients' access to these orphan drugs. These 37 drugs are also classified as orphan drugs used for leukemia treatment by the Food and Drug Administration (FDA).

Numerous studies have been performed to assess the cost-effectiveness of orphan drugs in leukemia treatment. This information could support the effort of reducing the financial burden for leukemia patients as well as other potential payers. Policymakers in Vietnam could also consider this costeffectiveness evidence to implement optimal pricing negotiations with suppliers or adjusting the health insurance coverage of leukemia patients.

This study was carried out to systematically review all cost-effectiveness analysis (CEA) studies of these 37 orphan drugs for leukemia treatment globally.

\section{MATERIALS AND METHODS}

This study conducted a systematic review on all CEA studies published till August 2021 on the National Center for Biotechnology Information (NCBI), SpringerLink, and Biomed Central, with the following search formula: ("costeffectiveness" or "cost-utility" or "cost analysis") AND ("drug name" or "orphan drug") AND "leukemia treatment". Corresponding to 37 leukemia drugs listed on the MOH's Orphan Drugs List, 37 different search formulas were formed and 2099 original studies were identified. After removing duplicates, there were 401 studies being screened then assessed for eligibility by two following main criteria: the full-text must be available and written in English, and the study must analyze the cost-effectiveness of studied orphan drugs used in leukemia treatment. As a result, 23 studies were selected in this systematic review. (Figure 1)

The results of this systematic review were presented by describing the distribution of selected studies by leukemia types, main characteristics of selected studies, CEA methods, and CEA findings. There were five major leukemia types identified, including acute lymphoblastic leukemia (ALL), acute myeloid leukemia (AML), acute promyelocytic leukemia (APL), chronic lymphocytic leukemia (CLL), and chronic myeloid leukemia (CML). The main characteristics of selected studies included leukemia type, treatment condition, payment perspective, clinical endpoint, and effectiveness measurement unit. Characteristics of CEA methods were described by model type, scenario analysis, sensitivity analysis, time-horizon, and discount rate. The CEA findings, including the ICER of each orphan drug and the comparison to the corresponding WTP threshold, were reported by leukemia types, along with information on the country, year of study, and sample size. 
All the cost currency values were converted to USD in 2021.

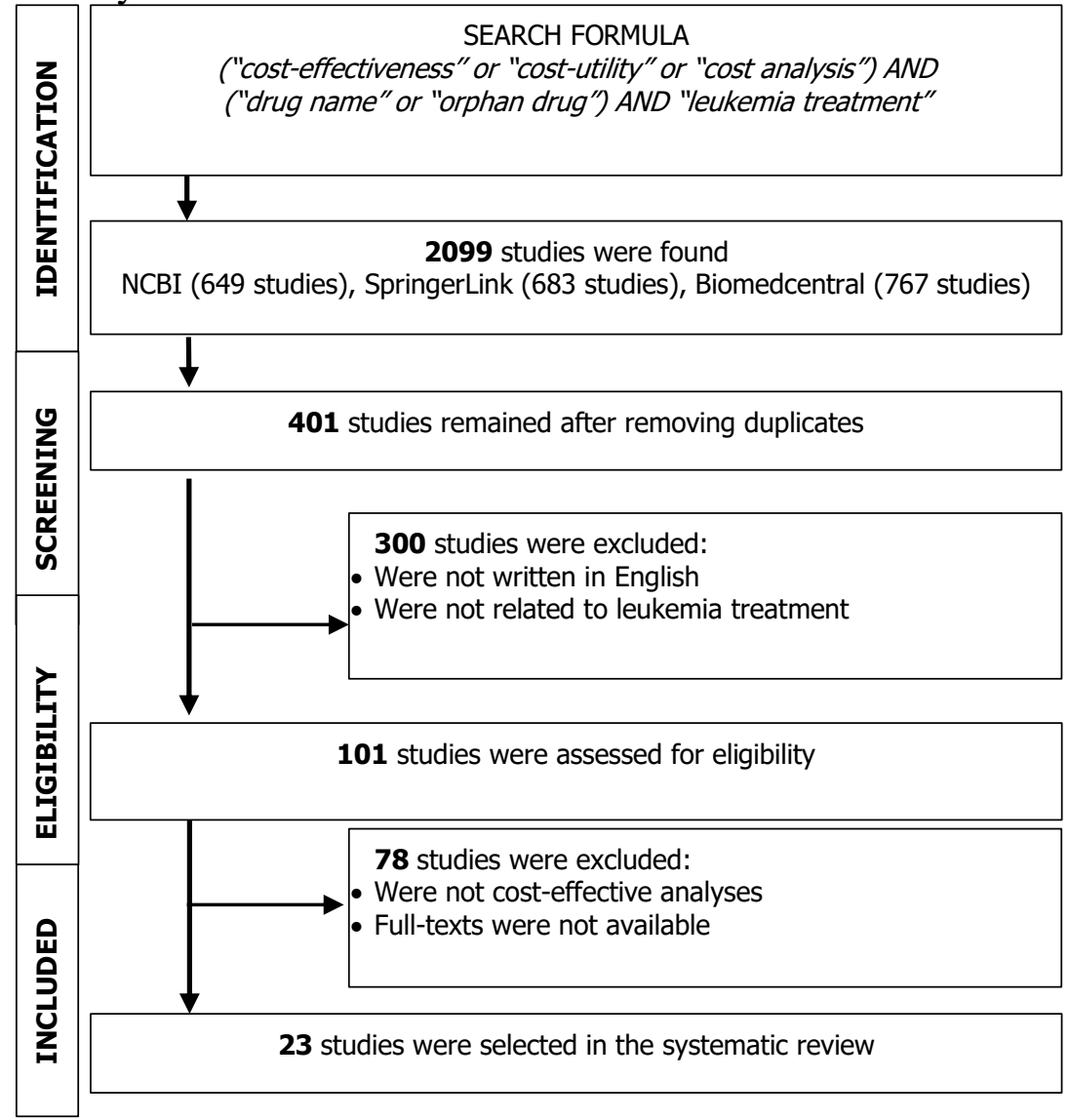

Figure 1. The systematic review study selection process following the PRISMA diagram

\section{RESULTS}

Orphan drugs applied in full-text CEA: Out of 37 orphan drugs, 24 orphan drugs were cost-effectiveness analyzed with available full-texts. According to the Vietnam MOH's Orphan Drug List, there were four drugs indicated for two different leukemia types, which were Imatinib (for ALL and CML), Ponatinib (for ALL and CML), Dasatinib (for ALL and CML), and
Venetoclax (for AML and CLL). This review showed that the cost-effectiveness of Venetoclax was analyzed for both AML and CLL, the cost-effectiveness of Imatinib and Dasatinib were analyzed for CML, while the cost-effectiveness of Ponatinib was not yet analyzed for any leukemia type. There were several studies analyzing the costeffectiveness of more than one orphan drug at a time $e^{[2-7,11-13,15,17,18,20-23]}$. (Table 1)

Table 1. Description of 37 orphan drugs of interest by leukemia types and the availability of CEA findings

\begin{tabular}{|c|c|c|}
\hline \multirow[b]{2}{*}{$\begin{array}{l}\text { Leukemia } \\
\text { types }\end{array}$} & \multicolumn{2}{|c|}{ Orphan drugs listed on the MOH's Orphan Drugs List $(n=37)$} \\
\hline & With available CEA findings $(n=24)$ & $\begin{array}{c}\text { Without available CEA } \\
\text { findings }(n=13)\end{array}$ \\
\hline $\begin{array}{c}\text { ALL } \\
(n=12)\end{array}$ & $\begin{array}{l}\text { Blinatumomab } \\
\text { PEGasparaginase }{ }^{[3,5]}, \text {,Erwinia L-asparaginase }{ }^{[3,5]} \text {, Inotuzumab ozogamicin } \\
\text { PE], } \\
\text { Clofarabine }\end{array}$ & $\begin{array}{l}\text { Nelarabine, Vincristine, } \\
\text { Methotrexate, Mercaptopurine, } \\
\text { *Ponatinib, *Imatinib, *Dasatinib }\end{array}$ \\
\hline
\end{tabular}




\section{VIETNAM MEDICAL JOURNAL №1\&2/2021}

\begin{tabular}{|c|c|c|}
\hline \multirow{2}{*}{$\begin{array}{l}\text { Leukemia } \\
\text { types }\end{array}$} & \multicolumn{2}{|c|}{ Orphan drugs listed on the MOH's Orphan Drugs List $(n=37)$} \\
\hline & With available CEA findings $(n=24)$ & $\begin{array}{c}\text { Without available CEA } \\
\text { findings }(n=13)\end{array}$ \\
\hline AML $(n=9)$ & $\begin{array}{l}\text { Cytarabine }{ }^{[12-15]}, \text { Daunorubicin } \\
\left.\text { Glasdegib }^{[14]}, 13,15\right] \\
\text { Venetoclax } \\
\text { Vemtuzumab ozogamicin }\end{array}$ & $\begin{array}{l}\text { Gilteritinib, Enasidenib, } \\
\text { Ivosidenib }\end{array}$ \\
\hline APL $(n=2)$ & Arsenic trioxide ${ }^{[17,18]}$, All-trans-retinoic-acid $[17,18]$ & \\
\hline $\begin{array}{c}\text { CLL } \\
(n=11)\end{array}$ & $\begin{array}{l}\text { Idelalisibib], Rituximab }{ }^{[7,11]} \text {, Ofatumumab }{ }^{[8]} \text {, Ibrutinib }{ }^{[9,} \\
\text { 10], Acalabrutinib }{ }^{[10]} \text {, Bendamustine }{ }^{[11]}, \text { Venetoclax }^{[11]}\end{array}$ & \begin{tabular}{|l|} 
Alemtuzumab, Duvelisib, \\
Fludarabine, Obinutuzumab
\end{tabular} \\
\hline CML $(n=7)$ & $\begin{array}{l}\text { Imatinib }[19,21,23], \text { Nilotinib }[20-22], \text { Dasatinib }^{[20-23]}, \\
\text { Interferon alfa-2a }\end{array}$ & *Ponatinib, Omacetaxine \\
\hline
\end{tabular}

Main characteristics of selected studies: Of 23 selected studies, $60.9 \%$ studied on relapsed/refractory patients, $39.1 \%$ conducted cost-analyses with a social perspective, and $47.7 \%$ used overall survival combined with progression-free survival (OS-PFS) as a clinical endpoint. Quality-adjusted life year (QALY) was used as an effectiveness measurement unit in $65.2 \%$ of the total selected studies, while life-year gained (LYG) and QALY - LYG were used in $4.4 \%$ and $30.4 \%$, respectively. There were 10 studies regarding lymphocytic leukemia $(43.5 \%)^{[2-11]}$ and 13 studies regarding myeloid leukemia $(56.5 \%)^{[12-24]}$. (Table 2)

Table 2. Main characteristics of 23 selected studies

\begin{tabular}{|c|c|c|c|}
\hline \multirow{2}{*}{ Charateristics of studies } & \multicolumn{2}{|c|}{ Total } & \multirow{2}{*}{ References } \\
\hline & $\mathbf{n}$ & $(\%)$ & \\
\hline \multicolumn{4}{|c|}{ Treatment condition } \\
\hline Untreated/ Newly diagnosed & 9 & (39.1) & {$[5,12,14-17,19,21,24]$} \\
\hline Relapsed/ Refractory & 14 & $(60.9)$ & {$[2-4,6-11,13,18,20,22,23]$} \\
\hline \multicolumn{4}{|c|}{ Payment Perspective } \\
\hline Patient & 8 & $(34.8)$ & {$[2,4,6,9,14,16,18,24]$} \\
\hline Provider & 4 & $(17.4)$ & {$[15,19,20,23]$} \\
\hline Social organization & 9 & (39.1) & {$[3,5,8,10-12,17,21,22]$} \\
\hline Not mentioned & 2 & $(8.7)$ & {$[7,13]$} \\
\hline \multicolumn{4}{|c|}{ Clinical endpoints } \\
\hline OS (Overall survival) & 4 & $(17.4)$ & {$[12,13,17,18]$} \\
\hline EFS (Event-Free Survival) & 2 & $(8.7)$ & {$[3,15]$} \\
\hline OS-PFS (Progression-Free Survival) & 11 & $(47.7)$ & {$[7-11,14,19,20,22-24]$} \\
\hline OS-EFS & 4 & $(17.4)$ & {$[2,4-6]$} \\
\hline OS-PFS-EFS & 1 & $(4.4)$ & [16] \\
\hline Others & 1 & $(4.4)$ & [21] \\
\hline \multicolumn{4}{|c|}{$\begin{array}{ll}\text { Effectiveness measurement unit } \\
\end{array}$} \\
\hline Quality-Adjusted Life Year (QALY) & 15 & $(65.2)$ & {$[2,4,5,9,10,12,14-16,18-21,23,24]$} \\
\hline Life-Year Gained (LYG) & 1 & $(4.4)$ & {$[3]$} \\
\hline QALY - LYG & 7 & $(30.4)$ & {$[6-8,11,13,17,22]$} \\
\hline \multicolumn{4}{|c|}{ Leukemia types } \\
\hline Acute lymphoblastic leukemia & 5 & $(21,7)$ & {$[2-6]$} \\
\hline Acute myeloid leukemia & 5 & $(21,7)$ & {$[12-16]$} \\
\hline Acute promyelocytic leukemia & 2 & $(8,7)$ & {$[17,18]$} \\
\hline Chronic lymphocytic leukemia & 5 & $(21,7)$ & [7-11] \\
\hline Chronic myeloid leukemia & 6 & $(26,2)$ & {$[19-24]$} \\
\hline
\end{tabular}


Characteristics of CEA methods: Of 23 selected studies, $52.1 \%$ used a partitioned survival model (PSM) and $39.1 \%$ used a deterministic sensitivity analysis (DSA) in combination with a probabilistic sensitivity analysis (PSA). (Table 3).

Table 3. Characteristics of CEA methods used in 23 selected studies

\begin{tabular}{|c|c|c|c|}
\hline \multirow[t]{2}{*}{ Characteristics of CEA methods } & \multicolumn{2}{|c|}{ Total } & \multirow[t]{2}{*}{ References } \\
\hline & $\mathbf{n}$ & $(\%)$ & \\
\hline \multicolumn{4}{|c|}{ Model type } \\
\hline Markov & 10 & $(43,5)$ & {$[5,7,9,12,15,17-19,21,23]$} \\
\hline Decision-tree & 1 & $(4,4)$ & [3] \\
\hline Partitioned survival model (PSM) & 12 & $(52,1)$ & {$[2,4,6,8,10,11,13,14,16,20,22,24]$} \\
\hline \multicolumn{4}{|c|}{ Scenario analysis } \\
\hline Scenario analysis applied & 17 & $(73,9)$ & {$[2,3,5,6,9-11,13-17,19,21-24]$} \\
\hline Scenario analysis not applied & 6 & $(26,1)$ & {$[4,7,8,12,18,20]$} \\
\hline \multicolumn{4}{|c|}{ Sensitivity analysis } \\
\hline Probabilistic Sensitivity Analysis (PSA) & 3 & (13) & {$[8,14,23]$} \\
\hline One Way Analysis (OWA) & 4 & $(17,4)$ & {$[3,9,12,19]$} \\
\hline Deterministic Sensitivity Analysis (DSA) & 1 & $(4,4)$ & [22] \\
\hline OW - PSA & 4 & $(17,4)$ & {$[15,16,20,24]$} \\
\hline PSA - DSA & 9 & $(39,1)$ & {$[2,4-7,10,11,13,17]$} \\
\hline Others/ Not mentioned & 2 & $(8,7)$ & {$[18,21]$} \\
\hline \multicolumn{4}{|c|}{ Time-horizon } \\
\hline$<10$ years & 10 & $(43,5)$ & {$[3,5,6,12-14,18,19,22,23]$} \\
\hline $10-15$ years & 1 & $(4,4)$ & {$[8]$} \\
\hline$>15$ years & 12 & $(52,1)$ & {$[2,4,7,9-11,15-17,20,21,24]$} \\
\hline \multicolumn{4}{|c|}{ Annual discount rate } \\
\hline $1-1,9 \%$ & 2 & $(8,7)$ & {$[14,19]$} \\
\hline $2-2,9 \%$ & 1 & $(4,4)$ & {$[6]$} \\
\hline $3-4 \%$ & 18 & $(78,2)$ & {$[2-5,7-11,13,15-17,20-24]$} \\
\hline Not mentioned & 2 & $(8,7)$ & {$[12,18]$} \\
\hline
\end{tabular}

Summary of CEA findings: Of 23 selected studies, 15 studies made a conclusion that their studied orphan drugs were cost-effective. $100 \%$ of the studies regarding acute lymphoblastic leukemia showed the orphan drugs of interest were cost-effective (ICER < WTP threshold); while about $50 \%$ of the studies regarding other leukemia types showed the orphan drugs of interest were not cost-effective (ICER > WTP threshold). Blinatumomab, Ibrutinib, Imatinib, and Venetoclax were the most common drugs of interest (9 studies, $39.1 \%$ ) and had a total cost less than the corresponding WTP threshold. (Table 4) 
Table 4. Summary of CEA findings from 23 selected studies

\begin{tabular}{|c|c|c|c|c|}
\hline $\begin{array}{l}\text { Country, Year } \\
\text { of study } \\
\text { (Sample size) }\end{array}$ & $\begin{array}{l}\text { Orphan drug of } \\
\text { interest }\end{array}$ & Comparator & $\begin{array}{c}\text { ICER } \\
\text { (USD/QALY) } \\
\text { USD/LYG) }\end{array}$ & $\begin{array}{l}\text { WTP } \\
\text { thresh } \\
\text { old } \\
\text { (USD) }\end{array}$ \\
\hline \multicolumn{5}{|c|}{ Acute Lymphoblastic Leukemia (ALL) } \\
\hline $\begin{array}{l}\text { USA 2017 } \\
(n=405)^{[2]}\end{array}$ & Blinatumomab* & Standard of Care & $\begin{array}{l}110,108 \\
\text { (USD/QALY) }\end{array}$ & $\begin{array}{l}150,00 \\
0\end{array}$ \\
\hline $\begin{array}{l}\text { The Netherlands } \\
2019(n=68)^{[3]}\end{array}$ & Erwinia asparaginase* & PEG asparaginase & 1,892 (USD/LYG) & \\
\hline $\begin{array}{l}\text { USA 2019 } \\
(n=731)^{[4]}\end{array}$ & Blinatumomab* & Inotuzumab ozogamicin & $\begin{array}{l}4,006-20,737 \\
\text { (USD/QALY) }\end{array}$ & $\begin{array}{l}150,00 \\
0\end{array}$ \\
\hline $\begin{array}{c}\text { UK } 2019(\mathrm{n}= \\
2,911)^{[5]}\end{array}$ & PEG asparaginase* & Native asparaginase & $\begin{array}{l}-130,753 \\
\text { (USD/QALY) }\end{array}$ & 27,800 \\
\hline \multirow[t]{2}{*}{$\begin{array}{l}\text { Japan 2020 } \\
(\mathrm{n}=228)^{[6]}\end{array}$} & \multirow[t]{2}{*}{ Tisagenlecleucel* } & Blinatumomab & $\begin{array}{l}18,723 \\
\text { (USD/QALY) } \\
19152 \\
\text { (USD/LYG) }\end{array}$ & \multirow[t]{2}{*}{67,500} \\
\hline & & Clofarabine & $\begin{array}{l}24,331 \\
\text { (USD/QALY) } \\
24,315 \\
\text { (USD/LYG) }\end{array}$ & \\
\hline \multicolumn{5}{|c|}{ Actute Myeloid Leukemia (AML) } \\
\hline $\begin{array}{c}\text { USA } 2014 \\
(n=1,000)^{[12]}\end{array}$ & Decitabine & Daunorubicin + Cytarabine & $\begin{array}{l}-433,756 \\
\text { (USD/QALY) }\end{array}$ & - \\
\hline $\begin{array}{l}\text { Spain } 2019(n= \\
717)^{[13]}\end{array}$ & $\begin{array}{l}\text { Midostaurin + Cytarabine } \\
\text { + Daunorubicin* }\end{array}$ & Cytarabine + Daunorubicin & $\begin{array}{l}46,782 \\
\text { (USD/QALY) } \\
39,424(\text { USD/LYG }\end{array}$ & 60,000 \\
\hline \multirow[t]{2}{*}{$\begin{array}{l}\text { Canada } 2020(\mathrm{n}= \\
255)^{[14]}\end{array}$} & \multirow[t]{2}{*}{ Glasdegib + Cytarabine* } & Cytarabine & $\begin{array}{l}65,048 \\
\text { (USD/QALY) }\end{array}$ & \multirow[t]{2}{*}{80,000} \\
\hline & & Azacitidine & $\begin{array}{l}65,129 \\
\text { (USD/QALY) }\end{array}$ & \\
\hline $\begin{array}{l}\text { Spain 2021 }(n= \\
280)^{[15]}\end{array}$ & $\begin{array}{l}\text { Gemtuzumab ozogamicin } \\
+ \text { + Cytarabine }+ \\
\text { Daunorubicin* }\end{array}$ & Cytarabine + Daunorubicin & $\begin{array}{l}29,043 \\
\text { (USD/QALY) }\end{array}$ & $\begin{array}{l}12,000- \\
36,000\end{array}$ \\
\hline $\begin{array}{l}\text { USA } 2021(n= \\
400)^{[16]}\end{array}$ & Venetoclax + Azacitidine $†$ & Azacitidine & $\begin{array}{l}\text { 260,343 } \\
\text { (USD/QALY) }\end{array}$ & $\begin{array}{l}150,00 \\
0\end{array}$ \\
\hline \multicolumn{5}{|c|}{ Acute Promyelocytic Leukemia (APL) } \\
\hline \multirow[t]{2}{*}{$\begin{array}{l}\text { USA 2015 } \\
(n=672)^{[17]}\end{array}$} & $\begin{array}{l}\text { All-trans-retinoic-acid + } \\
\text { Idarubicin }\end{array}$ & $\begin{array}{l}\text { All-trans-retinoic-acid + } \\
\text { Cytarabine }\end{array}$ & $\begin{array}{l}3,122 \\
\text { (USD/QALY) } \\
2,933 \text { (USD/LYG) }\end{array}$ & \multirow{2}{*}{$\begin{array}{l}50,000 \\
150,00 \\
0 \\
\end{array}$} \\
\hline & $\begin{array}{l}\text { Arsenic trioxide + All- } \\
\text { trans-retinoic-acid* }\end{array}$ & $\begin{array}{l}\text { All-trans-retinoic-acid + } \\
\text { Idarubicin }\end{array}$ & $\begin{array}{l}5,614 \\
\text { (USD/QALY) } \\
4,512 \text { (USD/LYG) }\end{array}$ & \\
\hline $\begin{array}{l}\text { Mexico 2020 } \\
(n=20)^{[18]}\end{array}$ & $\begin{array}{l}\text { Arsenic trioxide + All- } \\
\text { trans-retinoic-acid }\end{array}$ & $\begin{array}{l}\text { International Consortium on } \\
\text { Acute Promyelocytic } \\
\text { Leukemia* }\end{array}$ & $\begin{array}{l}\text { Italy: } 6,497 \\
\text { USA: } 19,133 \\
\text { Canada: } 17,123 \\
\text { (USD/QALY) }\end{array}$ & 7,060 \\
\hline \multicolumn{5}{|c|}{ Chronic Lymphocytic Leukemia (CLL) } \\
\hline Spain 2018 & Idelalisib + Rituximab* & Rituximab & 36,000 & 54,000 \\
\hline
\end{tabular}


No 1\&2/2021 VIETNAM MEDICAL JOURNAL

\begin{tabular}{|c|c|c|c|c|}
\hline $\begin{array}{l}\text { Country, Year } \\
\text { of study } \\
\text { (Sample size) }\end{array}$ & $\begin{array}{l}\text { Orphan drug of } \\
\text { interest }\end{array}$ & Comparator & $\begin{array}{c}\text { ICER } \\
\text { (USD/QALY) } \\
\text { USD/LYG) }\end{array}$ & $\begin{array}{c}\text { WTP } \\
\text { thresh } \\
\text { old } \\
\text { (USD) }\end{array}$ \\
\hline $\begin{array}{c}(\text { Not } \\
\text { mentioned })^{[7]}\end{array}$ & & & $\begin{array}{l}\text { (USD/QALY) } \\
17,694 \\
\text { (USD/LYG) }\end{array}$ & \\
\hline $\begin{array}{l}\text { UK 2017 } \\
(n=233)^{[8]}\end{array}$ & Ofatumumab & Best Supportive Care & $\begin{array}{l}181,483 \\
\text { (USD/QALY) } \\
88,323 \\
\text { (USD/LYG) }\end{array}$ & - \\
\hline $\begin{array}{c}\text { USA } 2018(n= \\
269)^{[9]}\end{array}$ & Ibrutinibt & Clorambucil & $\begin{array}{l}189,000 \\
\text { (USD/QALY) }\end{array}$ & $\begin{array}{l}150,00 \\
0\end{array}$ \\
\hline $\begin{array}{l}\text { UK } 2019(n= \\
61)^{[10]}\end{array}$ & Acalabrutinib† & Ibrutinib & $\begin{array}{l}85,912 \\
\text { (USD/QALY) }\end{array}$ & 69,350 \\
\hline \multirow[t]{4}{*}{$\begin{array}{l}\text { USA 2019 } \\
(n=389)^{[11]}\end{array}$} & Venetoclax + Rituximab* & Bendamustine + Rituximab & $\begin{array}{l}\text { 62,043 } \\
\text { (USD/QALY) } \\
46,016 \\
\text { (USD/LYG) }\end{array}$ & \multirow[t]{4}{*}{$\begin{array}{l}150,00 \\
0\end{array}$} \\
\hline & & Ibrutinib & Dominant & \\
\hline & & Ibrutinib + Rituximab & Dominant & \\
\hline & & Idelalisib + rituximab & Dominant & \\
\hline \multicolumn{5}{|c|}{ Chronic Myeloid Leukemia (CML) } \\
\hline $\begin{array}{l}\text { UK 2003 } \\
(n=497)^{[19]}\end{array}$ & Imatinibt & $\begin{array}{l}\text { Daunorubicin + Cytarabine + } \\
\text { Tioguanine }\end{array}$ & $\begin{array}{l}40,788-58,712 \\
\text { (USD/QALY) }\end{array}$ & - \\
\hline \multirow{2}{*}{$\begin{array}{c}\text { UK } 2011 \\
(\text { Not } \\
\text { mentioned) }{ }^{[20]}\end{array}$} & Nilotinib & \multirow[t]{2}{*}{ Interferon-a* } & $\begin{array}{l}\text { 145,533 } \\
\text { (USD/QALY) }\end{array}$ & \multirow[t]{2}{*}{41,700} \\
\hline & Dasatinib & & $\begin{array}{l}114,814 \\
\text { (USD/QALY) }\end{array}$ & \\
\hline \multirow[t]{3}{*}{$\begin{array}{c}\text { USA } 2015 \\
\text { (Not } \\
\text { mentioned) } \\
\text { [21] }\end{array}$} & $\begin{array}{l}\text { Imatinib } \rightarrow \\
\text { Chemotherapy/Stem Cell } \\
\text { Transplant }\end{array}$ & $\begin{array}{l}\text { Non-Tyrosine Kinase } \\
\text { Inhibitors }\end{array}$ & $\begin{array}{l}171,700 \\
\text { (USD/QALY) }\end{array}$ & \multirow[t]{3}{*}{$\begin{array}{l}122,75 \\
5\end{array}$} \\
\hline & $\begin{array}{l}\text { Imatinib } \rightarrow \text { Nilotinib } \rightarrow \text { Che } \\
\text { motherapy/Stem Cell } \\
\text { Transplant } \dagger\end{array}$ & $\begin{array}{l}\text { Imatinib } \rightarrow \\
\text { Chemotherapy/Stem Cell } \\
\text { Transplant }\end{array}$ & $\begin{array}{l}253,500 \\
\text { (USD/QALY) }\end{array}$ & \\
\hline & $\begin{array}{l}\text { Nilotinib } \rightarrow \text { Dasatinib } \rightarrow \\
\text { Chemotherapy/Stem Cell } \\
\text { Transplant }{ }^{\dagger}\end{array}$ & $\begin{array}{l}\text { Imatinib } \rightarrow \text { Nilotinib } \rightarrow \\
\text { Chemotherapy/Stem Cell } \\
\text { Transplant }\end{array}$ & $\begin{array}{l}445,100 \\
\text { (USD/QALY) }\end{array}$ & \\
\hline $\begin{array}{l}\text { USA 2017 } \\
(n=597)^{[22]}\end{array}$ & Nilotinib* & Dasatinib & $\begin{array}{l}7,031 \\
\text { (USD/QALY) } \\
-5,753 \\
\text { (USD/LYG) } \\
\end{array}$ & - \\
\hline $\begin{array}{c}\text { China } 2017(n= \\
6_{670}{ }^{[23]}\end{array}$ & Dasatinibt & Imatinib & $\begin{array}{l}58,989 \\
\text { (USD/QALY) }\end{array}$ & 22,455 \\
\hline \multirow[t]{2}{*}{$\begin{array}{l}\text { USA 2021 } \\
(n=1,802)^{[24]}\end{array}$} & \multirow[t]{2}{*}{ Bosutinib* } & Dasatinib & $\begin{array}{l}\text { 19,811 } \\
\text { (USD/QALY) }\end{array}$ & \multirow{2}{*}{$\begin{array}{l}50,000 \\
150,00 \\
0\end{array}$} \\
\hline & & Nilotinib & $\begin{array}{l}41,932 \\
\text { (USD/QALY) }\end{array}$ & \\
\hline
\end{tabular}

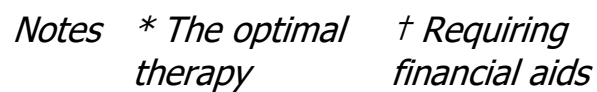




\section{DISCUSSION}

This study provided a systematic review of CEA studies of leukemia drugs listed on the Vietnam MOH's Orphan Drug List. 23 studies were reviewed, with the sample size varied from approximately 20 to 3000 leukemia patients with different distributions of gender and age. These studies were clinical trials that followed evidence-based principles and practice of publishing all information about the phase, sample size, and corresponding results on the United States National Library of Medicine. Most of the selected studies were conducted in developed countries such as the United States, the United Kingdom, and Spain (with 10, 5, 3 studies, respectively).

The conclusion of the cost-effectiveness of a drug depended on the WTP threshold used in each study. The WTP threshold of studies in the United States was the highest (150,000 USD) compared to the rest of countries in this review and was 20 times higher than that of Mexico (7,060 USD). Therefore, an orphan drug that was considered as cost-effective in the United States might not be considered as costeffective in other countries. In addition, the use of different effectiveness measurement units was another factor leading to different conclusions on the cost-effectiveness of studied orphan drugs. This systematic review recorded that ICER per QALY value was higher than ICER per LYG value ${ }^{[6-8,13,17,22]}$ and in two studies, the ICER per QALY value was double the ICER per LYG value ${ }^{[7}$, 8].

This review showed that conducting upto-date CEA studies of orphan drugs in leukemia treatment was necessary for the effort of reducing the financial burden.
Tisagenlecleucel was approved in Japan in 2019 for the ALL treatment and its CEA study was carried out in the same year, comparing this new intervention to the two first-line ALL treatment drugs, Blinatumomab and Clofarabine, by Wakase $\mathrm{S}$, et $a{ }^{[6]}{ }^{6]}$ The results showed that Tisagenlecleucel was highly cost-effective, with the ICER compared to Blinatumomab and Clofarabine were estimated at \$18,723/QALY and \$24,331/QALY respectively, which were all significantly lower than the Japanese WTP threshold of 68,297 USD. In another case, the conclusion on the cost-effectiveness of Ibrutinib therapy in the United States varied by year, which kept the healthcare system stay updated on the cost-effectiveness aspect of leukemia drug therapies. In 2018, Barnes JI, et al. calculated an ICER of 189,000 USD/QALY between Ibrutinib and Chlorambucil, which was greater than the corresponding WTP value, thus, suggested that payers should be offered financial aid in order to approach the better treatment ${ }^{[9]}$. In 2019, Huntington S.F., et al. showed evidence that Venetoclax + Rituximab was cost-effective compared to Ibrutinib, in the context of Venetoclax was approved for leukemia treatment by the FDA that year ${ }^{[11]}$.

To date, Vietnam does not have its own WTP threshold, therefore, it is recommended by the WHO to use a threshold of less than three times the national annual gross domestic product (GDP) per capita. This systematic review showed that most of the studied orphan drugs would not be considered as cost-effective in Vietnam if pure comparing the ICER values to the threshold of three times the Vietnam GDP per capita of 3,500 USD in 2021. However, taking into account that these studies were 
conducted in different populations and periods of time, information from this review should be only served as a reference to firstly understand the cost aspect of these orphan drugs worldwide, and then to conduct future research in Vietnamese populations. Only when aiding by high-quality and up-to-date CEA results then policymakers could make proper decisions on pricing negotiation or health insurance coverage in order to provide better care to leukemia patients.

\section{CONCLUSION}

The study provided an up-to-date review of the cost-effectiveness of 24 out of 37 orphan drugs for leukemia treatment listed on the Vietnam MOH's Orphan Drugs List. With considerably high costs, these orphan drugs could be a financial burden for leukemia patients and other potential payers such as the Vietnam Social Security. Therefore, financial aid policies are necessary to help patients timely access treatment, and better pricing negotiations with suppliers are needed to implement to reduce the financial burden of these orphan drugs.

\section{REFERENCES}

1. World Health Organization (2020), The Global Cancer Observatory, Available at https://gco.iarc.fr/today/data/factsheets/canc ers/36-Leukaemia-fact-sheet.pdf.

2. Delea TE, Amdahl J, Boyko D, et al. (2017). Cost-effectiveness of blinatumomab versus salvage chemotherapy in relapsed or refractory Philadelphia-chromosomenegative B-precursor acute lymphoblastic leukemia from a US payer perspective. J Med Econ 20(9): 911-922.

3. Kloos RQH, van Litsenburg RRL, Wolf $S$, et al. (2019). A cost-effectiveness analysis of Erwinia asparaginase therapy in children with acute lymphoblastic leukemia. Pediatric Blood \& Cancer 66(1): e27458.

4. Delea TE, Zhang $X$, Amdahl J, et al. (2019). Cost Effectiveness of Blinatumomab Versus Inotuzumab Ozogamicin in Adult Patients with Relapsed or Refractory B-Cell Precursor Acute Lymphoblastic Leukemia in the United States. Pharmacoeconomics 37(9): 1177-1193.

5. Hu X, Wildman KP, Basu S, et al. (2019). The cost-effectiveness of pegaspargase versus native asparaginase for first-line treatment of acute lymphoblastic leukaemia: a UK-based cost-utility analysis. Health Econ Rev 9(1): 40.

6. Wakase $\mathrm{S}$, Teshima $\mathrm{T}$, Zhang $\mathrm{J}$, et al. (2021). Cost-Effectiveness Analysis of Tisagenlecleucel for the Treatment of Pediatric and Young Adult Patients with Relapsed or Refractory B Cell Acute Lymphoblastic Leukemia in Japan. Transplant Cell Ther 27(3): 241.e241241.e211.

7. Casado LF, Hernández JÁ, Jarque I, et al. (2018). Cost-utility analysis of idelalisib in combination with rituximab in relapsed or refractory chronic lymphocytic leukaemia. European Journal of Haematology 100(3): 264-272.

8. Hatswell AJ, Thompson GJ, Maroudas PA, et al. (2017). Estimating outcomes and cost effectiveness using a single-arm clinical trial: ofatumumab for double-refractory chronic lymphocytic leukemia. Cost Eff Resour Alloc 15(1): 8.

9. Barnes JI, Divi V, Begaye A , et al. (2018). Cost-effectiveness of ibrutinib as first-line therapy for chronic lymphocytic leukemia in older adults without deletion 17p. Blood Advances 2(15): 1946-1956.

10. Vreman RA, Geenen JW, Hövels AM, et al. (2019). Phase I/II Clinical Trial-Based Early Economic Evaluation of Acalabrutinib for Relapsed Chronic Lymphocytic Leukaemia. Appl Health Econ Health Policy 17(6): 883-893.

11. Huntington SF, Torsten SM, Masaquel AS, et al. (2019). Cost-effectiveness of a 24-month 
fixed duration of venetoclax in combination with rituximab in relapsed or refractory chronic lymphocytic leukemia in the United States. Value in Health 22(2): 78-89.

12. Batty N, Yin Y, Wetzler M (2014). Decitabine is more cost effective than cytarabine and daunorubicin in elderly acute myeloid leukemia patients. Journal of Cancer Research \& Therapy 2(4): 68-73.

13. Arenaza A, Diez R, Esteve J, et al. (2019). Cost-Effectiveness of Midostaurin In The Treatment Of Acute Myeloid Leukemia With The FLT3 Mutation In Spain. Clinicoecon Outcomes Res 19(11): 683-694.

14. Hu Y, Charaan M, van Oostrum I, et al. (2021). The cost-effectiveness of glasdegib in combination with low-dose cytarabine, for the treatment of newly diagnosed acute myeloid leukemia in adult patients who are not eligible to receive intensive induction chemotherapy in Canada. J Med Econ 24(1): 150-161.

15. Mareque $M$, Montesinos $P$, Font $P$, et al. (2021). Cost-Effectiveness Analysis of Gemtuzumab Ozogamicin for First-Line Treatment of Patients with Cd-33 Positive Acute Myeloid Leukaemia in Spain. Clinicoecon Outcomes Res 21(13): 263-277.

16. Patel KK, Zeidan MA, Shallis MR, et al. (2021). Cost-effectiveness of azacitidine and venetoclax in unfit patients with previously untreated acute myeloid leukemia. Blood Advances 5(4): 994-1002.

17. Tallman M, Lo-Coco F, Barnes G, et al. (2015). Cost-Effectiveness Analysis of Treating Acute Promyelocytic Leukemia Patients With Arsenic Trioxide and Retinoic Acid in the United States. Clin Lymphoma Myeloma Leuk 15(12): 771-777.

18. Sergio RR, Lorena GT, Maria JDH, et al. (2020). Cost-effectiveness of the regimen proposed by the International Consortium on Acute Promyelocytic Leukemia for the treatment of newly diagnosed patients with Acute Promyelocytic Leukemia. Hematology, Transfusion and Cell Therapy.

19. Gordois A, Scuffham P, Warren E, et al. (2003). Cost-utility analysis of imatinib mesilate for the treatment of advanced stage chronic myeloid leukaemia. $\mathrm{Br} \mathrm{J}$ Cancer 89(4): 634-640.

20. Hoyle M, Rogers G, Moxham T, et al. (2011). Cost-Effectiveness of Dasatinib and Nilotinib for Imatinib-Resistant or Intolerant Chronic Phase Chronic Myeloid Leukemia. Value in Health 14(8): 1057-1067.

21. Rochau U, Kluibenschaedl M, Stenehjem D, et al. (2015). Effectiveness and CostEffectiveness of Sequential Treatment of Patients with Chronic Myeloid Leukemia in the United States: A Decision Analysis. Leuk Res Treatment 2015: 982395.

22. Li N, Yang X, Fan L, et al. (2017). Nilotinib versus dasatinib as second-line therapy in patients with Philadelphia chromosomepositive chronic myeloid leukemia in chronic phase who are resistant or intolerant to imatinib: a cost-effectiveness analysis based on real-world data. J Med Econ 20(4): 328336.

23. Bin W, Maobai L, Te L, et al. (2017). An economic analysis of high-dose imatinib, dasatinib, and nilotinib for imatinib-resistant chronic phase chronic myeloid leukemia in China: A CHEERS-compliant article. Medicine (Baltimore) 96(29): e7445.

24. Muresan B, Mamolo C, Cappelleri JC, et al. (2021). Cost-Effectiveness of Bosutinib for the Treatment of Adult Patients with Chronic Phase Chronic Myeloid Leukemia in the Second-Line Setting. Appl Health Econ Health Policy 1179-1896. 